\title{
Inequidades en salud de la primera infancia en el municipio de Andes, Antioquia, Colombia. Un análisis desde la epidemiología crítica*
}

\section{Inequities in Early Childhood Health in the Municipality of Andes, Antioquia, Colombia. A Critical Epidemiology Analysis}

\section{As desigualdades na saúde na infância na cidade de Andes, Antioquia, Colombia. Uma análise da epidemiologia crítica}

Fecha de recepción: 07-07-16 Fecha de aceptación: 06-09-16 Disponible en línea: 30-11-16 doi:10.11144/Javeriana.rgyps15-31.ispi

Cómo citar este artículo:

Franco-Cortés AM, Otálvaro-Castro GJ, Ochoa-Acosta E, Ramírez-Puerta S, Escobar-Paucar G, Agudelo-Suárez AA, Hernández-Paternina G, Manrique N. Inequidades en salud de la primera infancia en el municipio de Andes, Antioquia. Un análisis desde la epidemiología crítica. Rev. Gerenc. Polít. Salud. 2016; 15(31): 246-261. http://dx.doi.org/10.11144/Javeriana.rgyps15-31.ispi

\author{
Ángela María Franco-Cortés** \\ Gabriel Jaime Otálvaro-Castro*** \\ Emilia Ochoa-Acosta**** \\ Susana Ramírez-Puerta***** \\ Gloria Escobar-Paucar****** \\ Andrés A. Agudelo-Suárez $* * * * * *$ \\ Guido Hernández-Paternina******** \\ Néstor Manrique*********
}

\footnotetext{
Artículo de investigación. La financiación de la investigación estuvo a cargo del Proyecto Global Alianza por un Futuro Libre de Caries, convenio de alianza estratégica entre la Alcaldía de Andes-Antioquia, la Universidad de Antioquia y Colgate-Palmolive.

** Odontóloga, magíster en Epidemiología, Ph. D. en Ciencias Sociales. Niñez y Juventud. Profesora-investigadora de la Facultad de Odontología de la Universidad de Antioquia, Medellín, Colombia. Dirección: Calle 64 No. 52-59, Medellín, Colombia. Correo electrónico: franco.angelamaria@gmail.com

*** Odontólogo, epidemiólogo de campo, magíster en Educación, Ph. D. (c) en Salud Colectiva, Ambiente y Sociedad. Profesor-investigador de la Facultad Nacional de Salud Pública de la Universidad de Antioquia, Medellín, Colombia. Odontóloga, magíster en Educación y Desarrollo Humano, profesora-investigadora de la Facultad de Odontología de la Universidad de Antioquia y de la Universidad Cooperativa de Colombia Sede Envigado, Envigado, Colombia. Odontóloga, magíster en Epidemiología, profesora-investigadora de la Facultad de Odontología de la Universidad de Antioquia, Medellín, Colombia.

******* Odontopediatra, magíster en Salud Pública, profesora-investigadora de la Facultad de Odontología de la Universidad de Antioquia, Medellín, Colombia.

******** Odontólogo, especialista en Administración de Servicios de Salud, Ph. D. en Salud Pública, profesor asociado de la Facultad de Odontología de la Universidad de Antioquia, Medellín, Colombia.

${ }_{* * * * * * * * *}$ Médico cirujano, especialista en Gerencia de la Salud Pública, Ph. D. en Gerencia y Política Educativa, profesor de cátedra de la Facultad Nacional de Salud Pública de la Universidad de Antioquia, Medellín, Colombia. Gerente

$246^{*_{* * * * * * * * *} \text { Enfermero de la Universidad de Antioquia, coordinador de la Estrategia Atención Primaria en Salud, ESE Hospital }}$ San Rafael, municipio Andes.
} 


\section{Resumen}

En Colombia persisten inequidades en la salud de la primera infancia. Se realizó un estudio descriptivo para estimar las desigualdades en salud de la primera infancia del municipio de Andes, Antioquia. Las categorías teóricas utilizadas fueron la clase social y los modos de vida. Se hizo valoración clínica del crecimiento, del desarrollo y del estado de la dentición de 642 niños de cero a cinco años. Se encuestó a los adultos acompañantes para clasificar su posición de clase. El muestreo fue probabilístico en la zona urbana y por concentración en la rural. La población se clasificó en cinco fracciones de clase. Se observaron desigualdades en los modos de vida, en el desarrollo infantil y en la experiencia de caries dental; más graves en los niños de las familias subasalariadas. Las disparidades constituyen inequidades sociales, puesto que son producto de las condiciones de vida que les son impuestas a los grupos humanos de acuerdo con su posición social.

Palabras clave: clase social; modo de vida; primera infancia; situación de salud; inequidades

\section{Abstract}

Colombia recognizes health as a fundamental right, however persistent inequities in early childhood. The objective was to describe the situation of early childhood and health inequalities between social groups in the municipality of Andes-Antioquia. A descriptive study framed in critical epidemiology was conducted, included assessment of growth and development and the State of dentition of 642 children 0 to 5 years. The accompanying adult was also surveyed. The sampling was probabilistic in urban areas and for concentration in rural areas. The population was classified into five social groups. Indicators of nutritional status and dental caries experience behaved unevenly, being better in children from families of employees/independent with higher education and worse in the group of day laborers. Inequities in oral health and child development among children sub-salaried families and other groups are evident.

Keywords: social determinants of health; social class; health status; oral health; early childhood; inequalities

\section{Resumo}

Colômbia desigualdades na saúde persistem na primeira infância. Um estudo descritivo para estimar as desigualdades na saúde na primeira infância no município de Andes, Antioquia foi realizada. As categorias teóricas utilizadas eram de classe social e estilo de vida. Avaliação clínica de crescimento, desenvolvimento e estado de dentição de 642 crianças de zero a cinco anos foi feita. Os adultos acompanhantes foram entrevistados para classificar a sua posição de aula. A amostragem foi probabilística na concentração urbana e rural. A população foi classificada em cinco frações de classe. desigualdades no estilo de vida, o desenvolvimento da criança e da experiência de cárie dentária foram observadas; mais grave em crianças de famílias subasalariadas. As disparidades são as desigualdades sociais, uma vez que são o resultado das condições de vida que lhes são impostas grupos humanos de acordo com a sua posição social.

Palavras-chave: classe social; estilo de vida; infância; situação de saúde; desigualdades 
Ángela María Franco-Cortés • Gabriel Jaime Otálvaro-Castro • Emillia Ochoa-Acosta •

Susana Ramirez-Puerta • Gloria Escobar-Paucar • Andrés A. Agudelo-Suárez • Guido

Hernández-Paternina • Néstor Manrique

\section{Introducción}

En las últimas décadas los gobiernos de Colombia han afirmado su compromiso con el mejoramiento de las condiciones de vida y de salud de la primera infancia (1-3), y se ha abierto paso al reconocimiento de su condición de sujetos de derechos, apenas visible desde el final del siglo XX (1). El marco normativo nacional reconoce a la salud, a la seguridad social y a la alimentación equilibrada como derechos fundamentales de la niñez $(1,3)$.

De los programas y las estrategias que materializan las disposiciones normativas se pueden destacar la estrategia nacional De Cero a Siempre - la cual busca garantizar la atención integral a la primera infancia (3) así como los lineamientos nacionales del Plan Decenal de Salud Pública 2012-2021, el cual plantea la necesidad de la definición territorial de líneas de base e implementación de procesos y estrategias tendientes a la disminución de la morbilidad y la mortalidad neonatal y de la niñez (4).

A pesar de dicho desarrollo normativo, persisten inequidades regionales y entre grupos sociales en la situación de salud y bienestar de la primera infancia en Colombia, así como graves situaciones de vulneración de los derechos de niños y niñas. Continúan como problemáticas no superadas un conjunto de situaciones evitables como la mortalidad neonatal, las bajas coberturas de vacunación, la violencia intrafamiliar, el bajo peso al nacer, la desnutrición y la caries dental, entre otras (5).

En el orden departamental, la política de primera infancia en Antioquia (6,7) se propuso la configuración de entornos protectores, prácticas de cuidado y acciones de prevención a la vulneración de los derechos de los niños y las niñas. En dicho contexto, a partir del año 2013 se implementó la estrategia de atención integral a la primera infancia Buen Comienzo Antioquia, que pretende el desarrollo infantil temprano y reconoce al niño y la niña como sujetos de derechos (6). En este marco político y normativo, particularmente, el gobierno del municipio de Andes, Antioquia del periodo 2012-2015 se propuso en el Plan de Desarrollo Municipal favorecer el desarrollo infantil temprano, procurando un trabajo articulado entre diferentes actores sociales e institucionales, incluida la Universidad de Antioquia $(8,9)$.

Para una implementación más efectiva, estas políticas, programas y estrategias se apoyan en el análisis de los modos de vida de los niños y niñas y sus familias; por lo tanto, en el ámbito nacional y local se insiste en la necesidad de llevar a cabo este tipo de estudios. En consecuencia, se presentan los resultados de un estudio, el cual, enmarcado en la perspectiva teórica de la epidemiología crítica (10-17), se hizo con el objetivo de describir la situación de salud de la primera infancia del municipio de Andes, Antioquia y la distribución de las inequidades de dicha situación entre los diferentes grupos sociales que conforman el municipio, con el fin de que los resultados y el análisis aquí presentados constituyan una línea de base para la evaluación del impacto del proyecto Alianza por un futuro libre de caries-capítulo Colombia (AFLC/CC), en un horizonte de trabajo a doce años, a la vez que permita la orientación de las decisiones locales dirigidas a generar acciones concretas para el mejoramiento de las condiciones de vida y salud de la primera infancia del municipio (9).

Para la epidemiología crítica, la salud y la enfermedad no se distribuyen de manera aleatoria en los individuos y grupos de una sociedad, sino que son resultado de la distribución desigual del poder que se produce por las condiciones económicas y políticas 
concretas en las que se estructura y desarrolla la sociedad y tiene lugar la vida de los individuos y los grupos (10).

\section{Materiales y métodos}

Se realizó un estudio descriptivo transversal en la población de niños y niñas de cero a cinco años de edad residentes en el área urbana y rural (siete corregimientos) del municipio de Andes, Antioquia. La población total de niños y niñas menores de cinco años en el municipio (3525) se estimó con base en la información del programa de vacunación del hospital local y de la encuesta de selección de beneficiarios. Con un nivel de confianza del $95 \%$ y un error de muestreo del $2 \%$, se calculó un tamaño de muestra de 717 niños y niñas, de los cuales 453 serían de la zona rural, distribuidos proporcionalmente en los siete corregimientos, y 264 de la zona urbana. Finalmente, la población evaluada fueron 642 niños y niñas, 442 en la zona rural y 200 en la zona urbana. La diferencia entre la muestra calculada y la población evaluada se dio por las dificultades para el desplazamiento en la zona rural y, posiblemente, por una sobrestimación del número total de niños en la zona urbana, dado que las fuentes consultadas tenían datos variables y no concordantes. El porcentaje de no respuesta fue de cero.

Dada la dispersión geográfica, en la zona rural se contactó a los niños y a los cuidadores mediante convocatoria abierta hecha por las promotoras de salud de cada vereda, para que se concentraran en la escuela o el centro de salud más cercano, donde se realizó la recolección de la información. En la zona urbana fue posible hacer un muestreo probabilístico y polietápico; por lo tanto, los niños fueron contactados en sus viviendas.
El protocolo del estudio fue aprobado por el Comité de Ética de la Facultad de Odontología de la Universidad de Antioquia, mediante el acta $n^{\circ} 222013$ de noviembre del 2014. También fue aprobado por las directivas de la ESE Hospital San Rafael del municipio de Andes y por la Secretaría Municipal de Salud. Se diligenció consentimiento informado de los adultos responsables para autorizar la participación de los niños en el estudio, y se contó con el asentimiento de niños y niñas al momento de la evaluación.

Las categorías teóricas utilizadas para clasificar la población del municipio en grupos sociales fueron clase social y modos de vida. La primera de ellas se entiende como la característica estructural determinante del modo de vida de un conglomerado social que comparte la misma posición en el sistema de relaciones sociales, y que corresponde a un mismo tipo de inserción en el sistema económico, de la cual depende el tipo de actividad de sus miembros, sus formas de propiedad, sus relaciones técnicas en el trabajo y la forma y tipo de cuota de la riqueza social que disfrutan. Desde la clase social se determina el disfrute de derechos humanos y el acceso a los bienes de los que depende la calidad de la reproducción social $(10,14,16,17)$.

Los modos de vida se refieren a los patrones estructurados de vida de un cierto grupo social, según su inserción en las dinámicas de la reproducción social, tanto en sus momentos de producción como en aquellos de consumo. En este sentido, se asume que no existe un único modo de vida y que los diferentes estilos de vida, las concepciones y prácticas y los perfiles de enfermedad, discapacidad y muerte son característicos de los particulares modos de vida existentes en el municipio $(10,14,16,17)$.

Las técnicas para la recolección de información fueron la encuesta, el examen físico y el examen clínico bucal. Se utilizaron tres ins- 


\section{Ángela Maria Franco-Cortés • Gabriel Jaime Otálvaro-Castro • Emilia Ochoa-Acosta Susana Ramirez-Puerta • Gloria Escobar-Paucar • Andrés A. Agudelo-Suárez • Guido Hernández-Paternina • Néstor Manrique}

trumentos para el registro de la información: una encuesta para la clasificación de la clase social o fracción de clase a la que pertenecía el niño y su familia, así como a efectos de la caracterización de sus modos de vida; la escala abreviada del desarrollo, para valorar el estado de crecimiento y desarrollo de niños y niñas; $y$ un formulario para registrar la experiencia de caries dental. El personal responsable del diligenciamiento de los instrumentos fue capacitado previamente.

La encuesta para clasificar la clase social y la caracterización de los modos de vida se estructuró a partir del modelo teórico propuesto por Breilh $(10,14,16,17)$, el cual parte de cuatro indagaciones básicas sobre el principal responsable económico de la familia del niño: a) el lugar que ocupa esta persona en el proceso productivo, (b) las relaciones que tiene con los medios de producción, (c) el papel desempeñado en la organización del trabajo y (d) la cuota y forma de riqueza social que disfruta.

Los modos de vida se indagaron a través de preguntas relacionadas con: (a) las condiciones de trabajo y patrón laboral del responsable económico, (b) la calidad y disfrute de bienes de consumo en la familia, (c) la capacidad de producción de valores culturales que otorguen identidad y (d) la capacidad de empoderamiento y organización. Mediante la subcategoría estilo de vida se identificaron las variantes que son posibles respecto a un cierto modo de vida $(10,14,16,17)$.

En la zona rural en el $93 \%$ de los casos fue la madre como acompañante quien contestó la encuesta; en la zona urbana lo fue en el $84 \%$ de los casos. Los resultados se consolidaron en una tabla de combinaciones para cada grupo social que fue revisada registro por registro. Se calcularon frecuencias absolutas y relativas de los diferentes atributos del modo de vida. Se utilizó la razón de verosimilitud como prueba de independencia para valorar la significación estadística en la distribución de los atributos del modo de vida entre fracciones de clase.

Para valorar el crecimiento y el desarrollo se realizó una encuesta al cuidador acompañante y un examen físico a los niños participantes. Se registraron las variables: perímetro cefálico, talla y peso, con las cuales se construyeron las curvas de crecimiento con la ayuda del software Anthro y AnthroPlus de la Organización Mundial de la Salud (18). Se calcularon indicadores para: talla para la edad, peso para la talla, perímetro cefálico para la edad e índice de masa corporal (IMC) para la edad. Se calcularon frecuencias absolutas y relativas y se cruzaron los indicadores con la fracción de clase a la que pertenece el niño. Se realizaron pruebas Chi cuadrado de distribución de frecuencias, con el fin de observar posibles asociaciones entre variables.

Para la evaluación del desarrollo se utilizó la escala abreviada de desarrollo que toma en cuenta los parámetros de: motricidad gruesa, motricidad fina adaptativa, audición/ lenguaje y desarrollo personal/social. Para cada uno de estos parámetros se estableció la distribución de frecuencias según el grado de desarrollo (alerta, medio, medio alto y alto). Se realizaron pruebas Chi cuadrado de distribución de frecuencias con el fin de observar posibles asociaciones entre variables.

El examen bucal evaluó la experiencia de caries dental en los niños que tenían al menos un diente presente. Previamente al examen los niños fueron cepillados por el adulto acompañante. Los exámenes fueron realizados por dos odontólogos calibrados en el diagnóstico de caries dental con base en el Sistema Internacional de Detección y Evaluación de Caries para el registro de caries dental por superficie (19). Las superficies dentales se secaron con gasa y se realizó inspección visual con espejo bucal plano y sonda WHO 11.5B. El 
hallazgo de lesiones consistentes con el nivel 1 se sumó al nivel 2 (ICDAS Epi), debido a que las condiciones del trabajo de campo no garantizaban el secado de la superficie dental con aire $(19,20)$. Los exámenes se hicieron en presencia de un adulto acompañante y se diligenció remisión a los servicios de salud de los niños que requerían atención.

Los datos recolectados en el examen bucal fueron analizados en el programa SPSS ${ }^{\circledR}$ versión 18. Se exploraron diferencias en la experiencia de caries dental según la fracción social a la que pertenece el niño, a través de la prueba Chi-cuadrado y la prueba de rangos múltiples. Por medio de la prueba Kruskal-Wallis se evalúo la significancia de las diferencias entre las medianas. Se utilizó un nivel de significación estadística del $5 \%$.

\section{Resultados}

\section{Clases y fracciones de clase representadas y sus modos de vida}

La población estudiada fue agrupada, según las variables contempladas en el enfoque teórico asumido, en tres clases y cinco fracciones de clase, así: (i) los asalariados, que a su vez se dividían en dos fracciones sociales: los calificados, es decir, empleados o independientes, con formación profesional o técnica (5,8\%), y los de baja calificación, empleados sin título profesional o técnico $(21,5 \%)$; (ii) los pequeños productores, igualmente representados por dos fracciones de clase: los pequeños comerciantes $(5,9 \%)$ y los pequeños productores agrícolas $(2,5 \%)$; y (iii) los subasalariados, por lo general jornaleros, sin fracciones de clase claramente definidas y quienes agruparon a la mayoría de los responsables económicos de las familias $(64,3 \%)$. La distribución territorial señala que el $68,8 \%$ de las familias viven en la zona rural y el $31,2 \%$ en la urbana.
Los cinco grupos sociales tienen algunas características diferenciadoras (tabla 1). Un punto de partida es el lugar que ocupan en el proceso de trabajo. Las dos fracciones de la clase asalariada se distinguen por ser trabajadores formales con diferentes niveles de calificación; los pequeños productores por ser propietarios de tierra, maquinaria o mercadería y por su trabajo manual; los subasalariados se distinguen porque realizan trabajo manual de baja calificación, de manera informal y en condiciones laborales precarias. Se diferencian a su vez por el nivel de escolaridad alcanzado, el cual es más alto entre la fracción de asalariados calificados y la más baja la de los subasalariados.

Al revisar los patrones de consumo las diferencias son más sutiles (tabla 1). Con respecto a las desigualdades en los ingresos económicos, se presentan diferencias estadísticamente significativas entre los asalariados calificados y los subasalariados (valor $\mathrm{p}<0.001$ ). Los ingresos económicos son bajos en términos generales. Vale la pena anotar que más de dos tercios de los responsables económicos de la familia reciben menos de un salario mínimo en todas las fracciones de clase, exceptuando los asalariados calificados.

Los niveles de precariedad de los ingresos guardan relación con la proporción de familias que reciben algún tipo de subsidio del Estado, que funciona como medida compensatoria y llega a cobijar al $8 \%$ de las familias de los asalariados calificados, el $25 \%$ en los pequeños productores agrícolas y hasta el $40 \%$ en los subasalariados (valor $\mathrm{p}<0.001$ ).

La proporción de familias donde los ingresos fueron insuficientes para la compra de alimentos se triplica entre los asalariados calificados y los subasalariados (5 y $15 \%$, respectivamente, valor $\mathrm{p}<0.005)$. $\mathrm{La}$ proporción de familias que participan en 


\section{Ángela Maria Franco-Cortés • Gabriel Jaime Otálvaro-Castro • Emilia Ochoa-Acosta \\ Susana Ramirez-Puerta • Gloria Escobar-Paucar • Andrés A. Agudelo-Suárez • Guido \\ Hernández-Paternina • Néstor Manrique}

organizaciones sociales es baja en términos generales; cerca de tres cuartas partes de la población encuestada no se encuentran vinculadas a organizaciones sociales, excepto los pequeños productores agrícolas, donde está vinculación llega al 50\%.

Con respecto a la propiedad de la vivienda no se observan disparidades marcadas entre clases, aunque resulta llamativo que una tercera y una quinta parte de los asalariados no calificados y subasalariados, respectivamente, habiten viviendas prestadas, así como la mayor precariedad habitacional de los subasalariados. La vivienda en préstamo es una práctica usual en las zonas rurales, donde los trabajadores reciben en préstamo pequeñas parcelas con viviendas de uso temporal mientras se trabaja en la zona, lo que funciona como un mecanismo compensatorio de la precariedad del ingreso.

Tabla 1. Distribución de los niños y sus familias SEgún la fracción de Clase y los modos de Vida. Andes, ANTIOQUiA, 2014

\begin{tabular}{|c|c|c|c|c|c|}
\hline \multirow{2}{*}{$\begin{array}{l}\text { Fracción } \\
\text { de clase }\end{array}$} & \multirow{2}{*}{$\begin{array}{l}\text { Frecuencia } \\
\text { absoluta y } \\
\text { (relativa) }\end{array}$} & \multicolumn{4}{|c|}{ Modos de vida } \\
\hline & & $\begin{array}{l}\text { Sociodemográfico } \\
\text { y propiedad }\end{array}$ & $\begin{array}{l}\text { Posición } \\
\text { ocupacional }\end{array}$ & Consumo & $\begin{array}{l}\text { Soportes } \\
\text { colectivos }\end{array}$ \\
\hline $\begin{array}{l}\text { Asalariados } \\
\text { calificados } \\
\text { (empleados e } \\
\text { independientes) }\end{array}$ & $37(5.8 \%)$ & $\begin{array}{l}\text { El } 45.9 \% \\
\text { profesionales, el } \\
54,1 \% \text { técnicos o } \\
\text { tecnólogos. Zona } \\
\text { urbana (62\%). } \\
\text { Una persona } \\
\text { en situación de } \\
\text { desplazamiento. } \\
\text { El } 67,5 \% \text { son } \\
\text { hombres. No } \\
\text { propietarios de } \\
\text { locales, tierra o } \\
\text { maquinaria. }\end{array}$ & $\begin{array}{l}\text { Empleados } \\
\text { públicos (docentes, } \\
\text { personal auxiliar); } \\
\text { profesionales } \\
\text { o técnicos que } \\
\text { ejercen como } \\
\text { independientes } \\
\text { (ingenieros, } \\
\text { administradores de } \\
\text { negocio, asesores } \\
\text { comerciales); } \\
\text { empleados } \\
\text { de empresas } \\
\text { particulares. }\end{array}$ & $\begin{array}{l}\text { Vivienda: propia } \\
(21.6 \%) \text {, alquiler } \\
(64.9 \%) \text {, prestada } \\
(13.5 \%) \text {. Ingresos: } \\
\text { menos de un } \\
\text { SMLV*(16.2\%), } \\
\text { entre } 1 \text { y } 2 \text { SMLV } \\
(51.4 \%) \text {, entre } 2 \text { y } 4 \\
\text { SMLV (24.3\%), más } \\
\text { de } 4 \text { SMLV (8.1\%). } \\
\text { Reciben subsidios } \\
\text { estatales (8.1\%). Le } \\
\text { faltó de dinero en } \\
\text { el último mes para } \\
\text { comprar alimentos: } \\
\text { nunca o casi nunca } \\
\text { (94.6\%), siempre } \\
\text { (5.4\%). Aseguramiento } \\
\text { en salud: régimen } \\
\text { subsidiado (21.6\%), } \\
\text { régimen contributivo } \\
\text { y especial (78.4\%) }\end{array}$ & $\begin{array}{l}\text { El } 75.7 \% \\
\text { no participa } \\
\text { en ninguna } \\
\text { organización } \\
\text { social }\end{array}$ \\
\hline $\begin{array}{l}\text { Asalariados no } \\
\text { calificados }\end{array}$ & $\begin{array}{l}138 \\
(21.5 \%)\end{array}$ & $\begin{array}{l}\text { Distribución } \\
\text { homogénea entre } \\
\text { zona urbana y } \\
\text { rural. En situación } \\
\text { de desplazamiento } \\
(4.3 \%) \text {. Culminó } \\
\text { estudios básicos } \\
\text { secundarios } \\
(39.9 \%) \text { - máximo } \\
\text { nivel educativo } \\
\text { alcanzado. El } 77 \% \\
\text { son hombres. No } \\
\text { propietarios de } \\
\text { locales, tierra o } \\
\text { maquinaria. }\end{array}$ & $\begin{array}{l}\text { Empleados de } \\
\text { empresas públicas } \\
\text { o particulares. } \\
\text { Ocupaciones u } \\
\text { oficios como: } \\
\text { vigilantes, cajeras, } \\
\text { vendedoras, } \\
\text { conductores, } \\
\text { secretarias, oficios } \\
\text { varios, escoltas, } \\
\text { meseras, ebanistas, } \\
\text { operarios, } \\
\text { mecánicos, } \\
\text { empleados de } \\
\text { fábricas y del sector } \\
\text { de la construcción, } \\
\text { encargados de } \\
\text { fincas, militares, } \\
\text { jubilados y } \\
\text { cuidadoras/ } \\
\text { jardineras. }\end{array}$ & $\begin{array}{l}\text { Vivienda: propia } \\
(30.4 \%) \text {, alquiler } \\
(42 \%) \text {, prestada } \\
(21.7 \%) \text {. Ingresos: } \\
\text { menos de un SMLV } \\
(67.6 \%) \text {, entre } 1 \text { y } 2 \\
\text { SMLV }(25.4 \%) \text { más } \\
\text { de } 2 \text { SMLV ( } 8.6 \%) \text {. } \\
\text { Reciben subsidios } \\
\text { estatales (26.8\%). Le } \\
\text { faltó dinero en el último } \\
\text { mes para comprar } \\
\text { alimentos: siempre o } \\
\text { casi siempre (5.8\%). } \\
\text { Aseguramiento en salud: } \\
\text { régimen subsidiado } \\
\text { (44.9\%), régimen } \\
\text { contributivo y especial } \\
(52.9 \%), \text { no afiliados } \\
\text { a la seguridad social } \\
(2.2 \%) .\end{array}$ & $\begin{array}{l}\text { El } 84.1 \% \\
\text { no participa } \\
\text { en ninguna } \\
\text { organización } \\
\text { social }\end{array}$ \\
\hline
\end{tabular}




\begin{tabular}{|c|c|c|c|c|c|}
\hline \multirow{2}{*}{$\begin{array}{l}\text { Fracción } \\
\text { de clase }\end{array}$} & \multirow{2}{*}{$\begin{array}{l}\text { Frecuencia } \\
\text { absoluta y } \\
\text { (relativa) }\end{array}$} & \multicolumn{4}{|c|}{ Modos de vida } \\
\hline & & $\begin{array}{l}\text { Sociodemográfico } \\
\text { y propiedad }\end{array}$ & $\begin{array}{l}\text { Posición } \\
\text { ocupacional }\end{array}$ & Consumo & $\begin{array}{l}\text { Soportes } \\
\text { colectivos }\end{array}$ \\
\hline $\begin{array}{l}\text { Pequeños } \\
\text { comerciantes }\end{array}$ & $38(5.9 \%)$ & $\begin{array}{l}\text { Zona urbana } \\
(60.5 \%) \text {. Una } \\
\text { persona es víctima } \\
\text { de desplazamiento. } \\
\text { Completó } \\
\text { educación } \\
\text { secundaria } \\
(42.1 \%) \text {, el } \\
31.5 \% \text { no lo } \\
\text { hizo. El } 73.6 \% \\
\text { son hombres. } \\
\text { Poseen pequeñas } \\
\text { extensiones de } \\
\text { tierra y maquinaria } \\
\text { liviana. }\end{array}$ & $\begin{array}{l}\text { Propietarios de un } \\
\text { pequeño negocio } \\
\text { que incluye locales } \\
\text { y mercadería. Se } \\
\text { dedican al comercio. }\end{array}$ & $\begin{array}{l}\text { Vivienda: propia } \\
(42.1 \%) \text {, alquiler } \\
(42.1 \%) \text {. Ingresos: } \\
\text { menos de un SMLV } \\
(79 \%) \text {, entre } 1 \mathrm{y} \\
2 \text { SMLV }(18.4 \%) \text {. } \\
\text { Reciben subsidios } \\
\text { estatales (18.4\%). } \\
\text { Le faltó dinero en } \\
\text { el último mes para } \\
\text { comprar alimentos: } \\
\text { siempre o casi siempre } \\
\text { ( } 7.9 \%) \text {. Aseguramiento } \\
\text { en salud: régimen } \\
\text { subsidiado (55.3\%), } \\
\text { régimen contributivo y } \\
\text { especiales (36.8\%), no } \\
\text { afiliados ( } 7.9 \%) \text {. }\end{array}$ & $\begin{array}{l}\text { El } 81.6 \% \\
\text { no participa } \\
\text { en ninguna } \\
\text { organización } \\
\text { social }\end{array}$ \\
\hline $\begin{array}{l}\text { Pequeños } \\
\text { agricultores }\end{array}$ & $16(2.5 \%)$ & $\begin{array}{l}\text { Zona rural } \\
(81.3 \%) \text {. El } 68.8 \% \\
\text { no completó } \\
\text { la educación } \\
\text { secundaria. } \\
\text { El } 87.5 \% \text { son } \\
\text { hombres. } \\
\text { Propietarios } \\
\text { de pequeñas } \\
\text { extensiones de } \\
\text { tierra y maquinaria }\end{array}$ & $\begin{array}{l}\text { Campesinos } \\
\text { propietarios de } \\
\text { tierra y maquinaria } \\
\text { que laboran o } \\
\text { dirigen el trabajo } \\
\text { agrícola en su } \\
\text { parcela, en la } \\
\text { cual se produce } \\
\text { especialmente café. }\end{array}$ & $\begin{array}{l}\text { Vivienda: propia } \\
(68.8 \%) \text {. Ingresos: } \\
\text { menos de un SMLV } \\
(68,8 \%) \text {, más de } \\
\text { un salario mínimo } \\
(31.2 \%) \text {. Reciben } \\
\text { subsidios estatales } \\
(25 \%) \text {. Le faltó } \\
\text { dinero en el último } \\
\text { mes para comprar } \\
\text { alimentos: siempre o } \\
\text { casi siempre }(12.5 \%) \text {. } \\
\text { Aseguramiento en } \\
\text { salud: régimen } \\
\text { subsidiado }(81.3 \%) \text {, } \\
\text { régimen contributivo } \\
\text { y especiales }(18.8 \%) \text {. }\end{array}$ & $\begin{array}{l}\text { El } 50 \% \\
\begin{array}{l}\text { pertenece } \\
\text { a alguna } \\
\text { organización } \\
\text { social }\end{array}\end{array}$ \\
\hline Subasalariados & $\begin{array}{l}413 \\
(64.3 \%)\end{array}$ & $\begin{array}{l}\text { Zona rural } \\
(80.4 \%) \text {. Víctimas } \\
\text { de desplazamiento } \\
(3.6 \%) \text {. El } 40.9 \% \\
\text { no completó la } \\
\text { educación básica } \\
\text { primaria, el } 7.7 \% \\
\text { no tiene ningún } \\
\text { estudio, y el } \\
36.5 \% \text { no culminó } \\
\text { la secundaria. } \\
\text { El } 83.3 \% \text { son } \\
\text { hombres. } \\
\\
\text { No propietarios } \\
\text { de locales, tierra o } \\
\text { maquinaria. }\end{array}$ & $\begin{array}{l}\text { Realizan trabajo } \\
\text { manual de baja } \\
\text { calificación, de } \\
\text { manera informal } \\
\text { y en condiciones } \\
\text { laborales precarias. } \\
\text { Incluyen: } \\
\text { peones agrícolas } \\
\text { o pecuarios, } \\
\text { trabajadores por día } \\
\text { en oficios varios, } \\
\text { en el comercio o en } \\
\text { ventas; empleadas } \\
\text { domésticas, } \\
\text { conductores, } \\
\text { trabajadores de las } \\
\text { minas, vendedores } \\
\text { ambulantes, } \\
\text { ayudantes familiares } \\
\text { sin remuneración y } \\
\text { amas de casa. }\end{array}$ & $\begin{array}{l}\text { Vivienda: propia } \\
(32.7 \%) \text {, alquiler } \\
(26.2 \%) \text {, prestada } \\
(35.8 \%) \text {. El } 4.3 \% \\
\text { de las viviendas } \\
\text { son improvisadas o } \\
\text { ranchos campesinos. } \\
\text { Ingresos: menos de } \\
\text { un SMLV (45.3\%), } \\
\text { hasta un SMLV } \\
(48.7 \%) \text {. El } 60 \% \\
\text { no recibe subsidios } \\
\text { estatales. Le faltó } \\
\text { dinero en el último } \\
\text { mes para comprar } \\
\text { alimentos: siempre o } \\
\text { casi siempre (15.3\%). } \\
\text { Aseguramiento en } \\
\text { salud: régimen } \\
\text { subsidiado ( } 91.5 \%) \text {, } \\
\text { régimen contributivo y } \\
\text { especiales (5.8\%), no } \\
\text { afiliados a la seguridad } \\
\text { social (2.2\%). }\end{array}$ & $\begin{array}{l}\text { El } 70 \% \text { no } \\
\text { participa } \\
\text { en ninguna } \\
\text { organización } \\
\text { social }\end{array}$ \\
\hline
\end{tabular}

* SMLV = salario mínimo legal vigente. Para el año 2014 fue de $\$ 616000$ pesos. En dólares fue de USD \$258, 253 según el valor del cambio a 31 de diciembre: \$2392 pesos.

Fuente: elaboración propia 
El comportamiento del aseguramiento en salud no resulta concordante con respecto a la situación laboral, puesto que no toda la población con un trabajo formal o independiente se encuentra afiliada al régimen contributivo. En todas las fracciones se encontró población en el régimen subsidiado, siendo mayoritaria en todas las fracciones de clase, exceptuando los asalariados calificados.

\section{Crecimiento y desarrollo infantil}

Los indicadores del estado nutricional mostraron que el $66.7 \%$ de los niños presentó talla normal para la edad, el $24.5 \%$ estaba en riesgo de talla baja y un $8.8 \%$ tenía talla baja para la edad. Con relación al peso, el $64.5 \%$ de los niños/as tenía peso adecuado para la talla, el $12.5 \%$ tenía riesgo de peso bajo para la talla y el $4 \%$ presentaba desnutrición aguda, debido a que tenía peso bajo o muy bajo para la talla. El $12.7 \%$ de los niños tenía sobrepeso y el $6.4 \%$ obesidad. Según el perímetro cefálico para la edad, un $10.7 \%$ de los niños/as están en riesgo para el neurodesarrollo (mayor riesgo en niñas, $\mathrm{p}<0.05$ ).
La prevalencia de talla baja y riesgo de talla baja fue mayor en los niños y niñas de los subasalariados, en tanto que fue menor en los de los asalariados calificados. Se encontraron diferencias estadísticamente significativas en la distribución porcentual de la población en riesgo o con talla baja según fracción de clase, tanto para la muestra total $(\mathrm{p}<0.01)$, como para en caso de los niños $(\mathrm{p}<0.01)$ (figura 1). La delgadez o riesgo de delgadez fue más frecuente $(25 \%)$ entre los niños de los pequeños agricultores, aunque no se encontraron diferencias estadísticamente significativas entre las fracciones sociales.

Con respecto al desarrollo de los niños y las niñas de Andes, en términos generales, la mayoría se encuentran en niveles de desarrollo medio y medio-alto. Tanto en niños como en niñas el porcentaje más alto en situación de alerta se presentó en la dimensión motricidad fina (3.9 y $1.7 \%$, respectivamente).

\section{Experiencia de caries dental}

Para conocer la experiencia de caries se examinó a 623 niños y niñas de cero a cinco años con al menos un diente erupcionado;

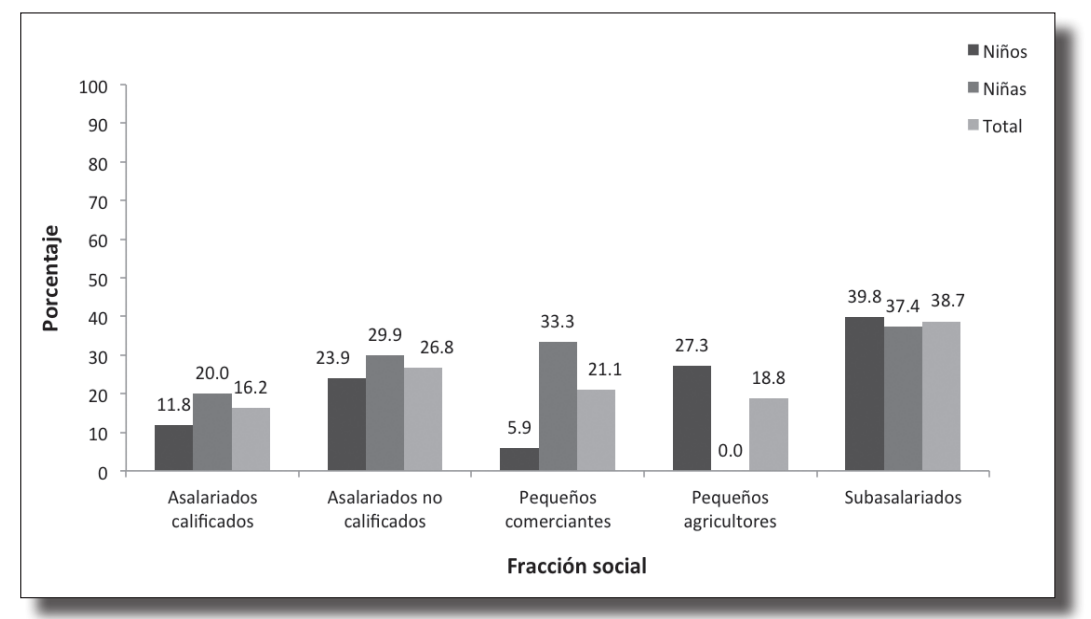

Figura 1. Prevalencia de talla baja para la edad o Riesgo de talla baja según fracción de Clase. Andes,

Fuente: elaboración propia 
434 de la zona rural $(69.7 \%)$ y 189 de la zona urbana del municipio (30.3\%). La experiencia modificada — que incluye tanto las lesiones iniciales y moderadas de caries sin cavidad que comprometa la dentina, como las lesiones que sí la comprometen ( $\mathrm{c}_{\text {ICDAS1-6 }}$ opd $\left.>0\right)(23)$ fue de $88.4 \%$, con diferencias significativas en la distribución según la zona de residencia (prueba Chi² de Pearson; $\mathrm{p}<0.006)$. La experiencia de caries, teniendo en cuenta solo las lesiones cavitarias $\left(\mathrm{c}_{\text {ICDAS5-6 }}\right.$ opd $>0$ ), fue de $38.2 \%$ (tabla 2).

La experiencia de caries modificada en el $91 \%$ de los hijos de los subasalariados es significativamente diferente cuando se la compara con la experiencia del $75 \%$ de los hijos de los asalariados calificados $(\mathrm{p}=0.007$, prueba exacta de Fisher). Las diferencias con otros grupos sociales no fueron significativas (tabla 2).

El cálculo del promedio $\mathrm{c}_{\mathrm{ICDAS5y}} \mathrm{opd}$ en el tercil de niños más afectados, denominado índice significante de caries ( $\mathrm{SiC}$ ) (21), se considera una forma de valorar la inequidad en la distribución de la caries dental. El SiC para la muestra de niños estudiada fue $4.3( \pm 3.0) ; 4.4( \pm 3.2)$ en la zona rural y $3,9( \pm 2.5)$ en la urbana. Esto significa que mientras en general cada niño tiene 1.5 dientes afectados por caries, aquellos que están más afectados tienen en promedio 4.3 dientes comprometidos $(\mathrm{p}<0.05)($ tabla 3$)$.

Las diferencias entre el SiC y el $\mathrm{c}_{\text {ICDAS5y6 }}$ opd son marcadas dentro de las fracciones de clase. Mientras en los hijos de los asalariados calificados la diferencia es de dos dientes, en los niños que provienen de familias de subasalariados es de tres, y en las de los pequeños agricultores es de cuatro dientes (tabla 3 ).

\section{Discusión y conclusiones}

La salud y la enfermedad constituyen un producto social que se presenta en los grupos humanos de manera desigual, de acuerdo con la manera como se distribuyen los recursos de poder en la sociedad (10). De ahí el interés del estudio por entender las características de los patrones grupales de vida.

Con respecto a la configuración de las clases sociales identificadas en el estudio, es preciso señalar que no quedaron representadas fracciones de la clase empresarial, lo que se explica porque, en términos generales, el

Tabla 2. Experiencia de Caries dental según fracción de Clase. Andes, Antioquia, 2014

\begin{tabular}{|c|c|c|c|c|c|c|c|c|}
\hline \multirow{3}{*}{ Fracción de clase } & \multicolumn{4}{|c|}{ Experiencia* } & \multicolumn{4}{|c|}{ Experiencia modificada $\dagger$} \\
\hline & \multicolumn{2}{|c|}{ Sí } & \multicolumn{2}{|c|}{ No } & \multicolumn{2}{|c|}{ Sí } & \multicolumn{2}{|c|}{ No } \\
\hline & $\mathbf{n}$ & $\%$ & $\mathbf{n}$ & $\%$ & $\mathbf{n}$ & $\%$ & $\mathbf{n}$ & $\%$ \\
\hline Asalariados calificados & 11 & 30,6 & 25 & 69.4 & 27 & $75.0^{a}$ & 9 & 25.0 \\
\hline Asalariados no calificados & 54 & 40.6 & 79 & 59.4 & 115 & 86.5 & 18 & 13.5 \\
\hline Pequeños comerciantes & 14 & 36.8 & 24 & 63.2 & 32 & 84.2 & 6 & 15.8 \\
\hline Pequeños agricultores & 5 & 31.3 & 11 & 68.8 & 13 & 81.3 & 3 & 18.8 \\
\hline Subasalariados & 154 & 38.5 & 246 & 61.5 & 364 & $91.0^{a}$ & 36 & 9.0 \\
\hline Total & 238 & 38.2 & 385 & 61.8 & 551 & 88.4 & 72 & 11.6 \\
\hline
\end{tabular}

* Porcentaje de niños/as con $\mathrm{c}_{\text {ICDAS } 5 \text { y } 6}$ opd $>0$.

$\dagger$ Porcentaje de niños/as con $\mathrm{c}_{\text {ICDAS 1- } 6}$ opd $>0$.

a (p 0.007 - prueba exacta de Fisher)

Fuente: elaboración propia 


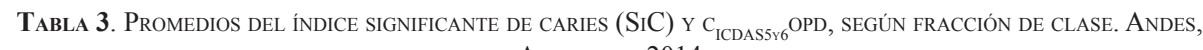
Antioquia, 2014

\begin{tabular}{|l|c|c|c|c|}
\hline \multirow{2}{*}{ Fracción de clase } & \multicolumn{2}{|c|}{ SiC } & \multicolumn{2}{c|}{$\mathbf{c}_{\text {ICDAS56 }}$ opd } \\
\cline { 2 - 5 } & Promedio & DE & Promedio & DE \\
\hline Asalariados calificados & 3.3 & 3.0 & 1.1 & 2.3 \\
\hline Asalariados no calificados & 4.4 & 2.8 & 1.6 & 2.6 \\
\hline Pequeños comerciantes & 2.9 & 2.3 & 1.0 & 1.9 \\
\hline Pequeños agricultores & $5.6^{a}$ & 4.9 & $1.8^{b}$ & 3.7 \\
\hline Subasalariados & $4.3^{b}$ & 3.1 & $1.5^{b}$ & 2.7 \\
\hline Total & 4.3 & 3.2 & 1.5 & 2.6 \\
\hline
\end{tabular}

Diferencia a y b $(\mathrm{p}<0.05)$

Fuente: elaboración propia

lugar de residencia permanente de esta clase social no es el propio municipio, aun cuando allí estén algunas de sus propiedades productivas, sitios de recreo y de descanso temporal.

Las tres clases sociales que se identificaron —asalariados, pequeños propietarios y subasalariados-y las cinco fracciones que las componen, se diferencian por los atributos predominantes de las formas de propiedad, el control/autonomía en el proceso de trabajo y la forma/cantidad de riqueza social que disfrutan. De ahí se derivan otros rasgos de sus modos de vida que los distinguen, particularmente según los niveles de calificación laboral, la formalidad del empleo y la escolaridad. Las disparidades en todos estos aspectos entre los asalariados de mayor calificación - fracción de profesionales y técnicos-y las demás fracciones son marcadas.

$\mathrm{Al}$ revisar las desigualdades sociales entre las clases representadas se puede concluir que las fracciones de clase representadas exponen una parte importante de la heterogeneidad de la sociedad andina, aun cuando no se trate de posiciones de clase polarizadas. De hecho, en algunos atributos presentan rasgos similares, tales como los bajos ingresos, la propiedad de las viviendas que habitan o la participación en organizaciones sociales.
Los resultados permiten identificar la manera como se expresan unas posiciones sociales, con modos de vida dispares entre sí y acceso diferencial a recursos de poder y oportunidades, en una sociedad definida por el peso de lo rural y la economía cafetera. En el contexto analizado, la producción de café — que representa el $73 \%$ del empleo rural- es el elemento determinante del ciclo de vida campesina y de la movilidad de la población en la región, lo cual está determinado por los periodos de crisis/bonanza que se presentan en el contexto nacional y en el internacional $(22,23)$. A pesar del estancamiento de la producción cafetera registrado desde el año 2005 y la diversificación de los usos del suelo hacia la ganadería extensiva, la minería y el turismo, en el municipio de Andes prevalece la vocación agrícola y cafetera en particular (24).

Al revisar las características del modo de vida de los grupos sociales andinos, en una población estudiada en la cual sus dos terceras partes habitan territorios rurales, se constatan importantes niveles de desigualdad social y precariedad en las condiciones de vida y reproducción social. Esto es similar al reporte oficial departamental y nacional, el cual caracteriza a la región suroeste como una zona de grandes desigualdades, de una pobreza estructural y de difícil superación, producto de una alta concen- 
tración de la tierra, la cual a su vez es resultado de la ocupación histórica del territorio y de los procesos de colonización agraria y asentamiento poblacional (24). La región suroeste es una de las más desiguales del departamento, el cual a su vez es uno de los más desiguales del país: el $92 \%$ de los predios son pequeñas parcelas de menos de veinte hectáreas y el indicador Gini de concentración de la distribución de la tierra es superior a 0.7 (24), muy superior al promedio nacional. De acuerdo con el Censo Nacional Agropecuario (CNA)-2014, el 45\% de la población residente en el área rural dispersa se encontró en condición de pobreza multidimensional (25).

Al analizar el periodo 2007-2011 se constató la disminución de los indicadores de calidad de vida y el aumento de las desigualdades entre la población rural y la población urbana en la subregión suroeste, producto de la crisis cafetera nacional (24). La relación entre crisis económica y salud ha sido estudiada desde la década de los treinta (26), en momentos en que se hicieron evidentes los efectos de la inestabilidad y la recesión económica en la salud de las personas. Con el final del siglo XX se han estudiado los efectos de los periodos de crisis económica en la profundización de las inequidades en salud, y se ha constatado cómo suelen ir acompañados de incrementos de la mortalidad en la población general y en algunos subgrupos como la infancia, sobre todo en países con menos recursos (27).

Los impactos de la crisis cafetera de la última década se reflejan en la alta proporción de población en estado de miseria y pobreza. De acuerdo con el análisis de situación de salud municipal, la miseria alcanza al 7.6\% de la población y es más alta en el área rural (9.2\%) que en la urbana (5.7\%); el 30\% de la población sobrevive con necesidades básicas insatisfechas y su distribución es desigual entre la población rural (38.2\%) y la urbana (21.9\%) (22).
Las brechas de equidad entre lo rural y lo urbano en el país han sido documentadas (28), y se destaca cómo a pesar del crecimiento económico sostenido de la última década, la población rural sigue siendo más pobre que la urbana, tiene menores oportunidades — tanto económicas como sociales-y menos acceso a los servicios del Estado, lo que repercute directamente en su calidad de vida y en las oportunidades para su desarrollo. Los hallazgos del CNA-2014 refuerzan la evidencia, pues constatan que el 23\% de hogares del área rural dispersa se encontró en condición de analfabetismo, el $82 \%$ presentó bajo logro educativo, el $57 \%$ de no cuenta con servicio de acueducto y el $94 \%$ no tiene acceso a alcantarillado (25).

En el caso particular de la primera infancia, persisten grandes brechas de inequidad territorial, en la medida en que los niños y las niñas que habitan en contextos rurales tienen la mitad de probabilidades que los de las zonas urbanas de vivir en casas con servicios públicos de energía, acueducto, alcantarillado y recolección de basuras, lo que conlleva una mayor probabilidad de mortalidad, desnutrición infantil y deterioro cognitivo (29).

Las condiciones materiales de vida que se derivan de la posición social de las personas definen sus posibilidades de desarrollo humano integral, así como las disparidades entre los grupos sociales. Los hallazgos respaldan esta tesis, puesto que demuestran de modo consistente cómo los indicadores de crecimiento, desarrollo infantil y salud bucal de los niños y niñas andinas resultan desiguales entre las fracciones de clase social. De esta manera, los mejores valores corresponden a los asalariados calificados y los peores a los subasalariados. Este hallazgo coincide con los resultados de la Encuesta Nacional de Situación Nutricional (30), en la cual los peores resultados se presentan en familias numerosas, de bajo nivel educativo, 
con necesidades básicas insatisfechas y pertenecientes a grupos étnicos minoritarios, así como con los hallazgos del CNA-2014 (25).

Los resultados sobre la experiencia de caries en los niños y en las niñas que se estudiaron también dan cuenta de desigualdades entre clases sociales: mientras que el porcentaje de niños y niñas afectados en familias de asalariados calificados es del $75 \%$, en los subasalariados llega al $91 \%(\mathrm{p}=0.007)$, lo que concuerda con los hallazgos de otros estudios que señalan que la caries dental es más frecuente en población que tiene baja escolaridad, está excluida del aseguramiento en salud y sus ingresos son bajos (31-37). Una mayor escolaridad puede desempeñar un doble papel: de un lado, más conocimientos que empoderen a las personas para participar del cuidado de la salud, y de otro lado, facilitar una mejor inserción en el trabajo, una mejor ocupación y con ello mayores ingresos para adquirir bienes y servicios.

Ahora bien, las diferencias encontradas en el promedio $\mathrm{C}_{\text {ICDAS5y6 }}$ opd y en el SiC, entre los pequeños agricultores y los subasalariados, hablan aún más de las inequidades y señalan, como en otros estudios, que si bien el porcentaje de personas libres de caries se ha incrementado en todos los grupos sociales (38), no ha ocurrido tanto así en el grupo de niños menores de seis años (39), lo que va acompañado de un aumento en la polarización de la enfermedad, porque una cantidad mayor de ella se concentra en pequeñas proporciones de niños con las peores condiciones socioeconómicas $(40,41)$. Esto es importante porque la sola medida de la experiencia tiene el riesgo de enmascarar las inequidades, ya que un resultado es el del número de niños afectados y otro distinto es cuánta enfermedad tienen $(42,43)$ y porque puede sugerir la orientación de medidas complementarias de política dirigidas a los grupos de mayor fragilidad social y exposición (44).
Pese a que los resultados demuestran ser consistentes en términos generales, también es importante destacar algunas limitaciones del estudio. Inicialmente, para recordar las restricciones del muestreo en la zona rural, pero también para reconocer que el enfoque teórico no fue desarrollado en toda su amplitud y quedó sin trabajar la dimensión simbólica de la clase social, así como otras dimensiones relevantes del modo de vida, entre ellas la dimensión cultural y las relaciones ecosistémicas. Tampoco fue explorada a profundidad la relación entre los modos de vida y las conductas individuales, lo que resulta importante para entender mejor el efecto del contexto en la toma de decisiones individuales. Algunas limitaciones de orden metodológico tuvieron que ver con el posible efecto de cohorte, propio de los estudios transversales, así como la limitación en la utilización de indicadores únicos de salud para develar procesos complejos como las inequidades sociales.

No obstante las limitaciones, la perspectiva teórica permitió reconocer diferencias en la posición social de la cual derivan modos de vida distintos, a partir de lo cual adquiere mayor sentido la explicación de las desigualdades en algunos indicadores de salud y la valoración de estas como inequidades sociales.

El estudio permite concluir que se presentan desigualdades en los modos de vida y los indicadores de salud infantil, cuyo origen no tiene relación con las decisiones individuales de las personas o familias; todo lo contrario, son el producto de condiciones materiales de vida que les son impuestas por su posición social, es decir, están determinados socialmente $(41,43,45,46)$. Estas condiciones deterioran y limitan el goce pleno de la salud, son evitables e injustas, y por tanto se convierten en inequidades sociales. El estudio de las desigualdades-inequidades sociales resulta prioritario en la agenda político-académica 
del país, en la medida en que las metas nacionales y globales se proponen la reducción de las desigualdades en salud (47-49).

Los resultados del estudio refuerzan el llamado a prestar mayor atención a lo rural, para reconocer las necesidades y los derechos de esta población, lo que cobra relevancia en el contexto de la necesidad de avanzar en una política integral de desarrollo rural, como condición de la construcción de una paz con mayores niveles de equidad y justicia social. En dicha política de desarrollo rural resulta indispensable una apuesta por la implementación de estrategias de salud rural que incorporen el desarrollo de infraestructura para la atención a la enfermedad y, especialmente, que se articulen con las políticas orientadas a mejorar las condiciones de vida y ampliar las oportunidades de desarrollo social de los habitantes rurales.

En esta vía resulta pertinente el llamado de la Comisión de Determinantes Sociales de la Salud con respecto a las recomendaciones para la acción en la primera infancia, llamado este que convoca a los gobiernos a implementar un conjunto integral de programas y servicios de calidad destinados a mejorar las condiciones de desarrollo de la primera infancia que tengan cobertura universal e incluyan a los niños, las madres y otros cuidadores, independientemente de su capacidad de pago, que promuevan la equidad de género y fomenten la participación comunitaria (49).

\section{Agradecimientos}

Los autores desean agradecer a las autoridades del municipio de Andes, al personal del hospital San Rafael, promotoras de salud y del servicio odontológico, y a los habitantes del municipio que participaron entusiasta y voluntariamente en este estudio. A la doctora Miryam Bastidas Acevedo, médica pediatra del Departamento de Pediatría Social de la Universidad de Antioquia, por su invaluable orientación y colaboración en el diseño y ejecución del proyecto. A la estrategia de sostenibilidad 2016-2017 del Comité para el Desarrollo de la Investigación (CODI) de la Universidad de Antioquia.

\section{Referencias bibliográficas}

1. Constitución Política de la República de Colombia de 1991.

2. República de Colombia, Ley 1098 del 2006, Código de la Infancia y la Adolescencia.

3. República de Colombia. Estrategia Nacional de Atención Integral a la Primera Infancia de Cero a Siempre. Bogotá, 2012.

4. República de Colombia, Ministerio de Salud y Protección Social. Resolución 1841, por la cual se adopta el Plan Decenal de Salud Pública 2012-2021. La salud la construimos todos. Bogotá, 2013.

5. Fondo de las Naciones Unidas para la Infancia (Unicef). Situación de la infancia en Colombia [Internet]. Disponible en: http://www.unicef.org/ argentina/spanish/educacion_Libro_primera_infancia.pdf

6. Gobernación de Antioquia. Plan de Desarrollo Departamental de Antioquia 2012-2015 "Antioquia la más Educada”. Medellín, 2012.

7. Gobernación de Antioquia. Estrategia de Atención Integral a la Primera Infancia: Buen Comienzo Antioquia. Bogotá, 2015.

8. Alcaldía de Andes. Plan de Desarrollo Municipal 2012-2015 "Juntos Construyendo Futuro". Andes, 2012. Disponible en: http://www.andes-antioquia.gov. co/apc-aa-files/32613564616231633066313831656238/ PROYECTO_DE_PLAN_DE_DESARROLLO.pdf

9. Alcaldía de Andes, Universidad de Antioquia, Colgate-Palmolive. Convenio de Alianza Estratégica 3230. Medellín, agosto del 2012.

10. Breilh J. Epidemiología crítica. Ciencia emancipadora e interculturalidad. Buenos Aires: Lugar Editorial; 2003.

11. Breilh J, Granda E. Investigación de la salud en la sociedad, guía pedagógica sobre un nuevo enfoque del método. Quito: Ediciones CEAs; 1980.

12. Breilh J. Epidemiología, economía política y salud. Bases estructurales de la determinación social de la salud, séptima edición. Quito: Universidad Andina Simón Bolívar, Corporación Editora Nacional; 2010.

13. Breilh J. Curso Internacional Determinantes Sociales de la Salud, conceptualización y estrategias de intervención. Santiago de Cali: Pontificia Universidad Javeriana; 2009.

14. Breilh J. Breve recopilación sobre operacionalización de la clase social para encuestas en la 
investigación social. Quito: Centro de Estudios y Asesorías en Salud; 1989.

15. Breilh J. Una perspectiva emancipadora de la investigación y acción, basada en la determinación social de la salud. En: Asociación Latinoamericana de Medicina Social. Taller Latinoamericano sobre Determinantes Sociales de la Salud [Internet]. México, D. F.: Alames; 2008. Disponible en http://repositorio. uasb.edu.ec/bitstream/10644/3413/1/Breilh,\%20JCON-119-Una\%20perspectiva\%20em.pdf

16. Breilh J. Las tres "S" de la determinación de la vida. 10 tesis hacia una visión crítica de la determinación social de la salud. Brasil: Cebes; 2010.

17. Breilh J. La determinación social de la salud como herramienta de transformación hacia una nueva salud pública (salud colectiva). Revista Facultad Nacional de Salud Pública. 2013, 31:13-27.

18. Organización Mundial de la Salud (OMS). Manual WHO Anthro para computadoras personales. Software para evaluar el crecimiento y desarrollo de los niños del mundo [Internet]. Ginebra: OMS; 2009. Disponible en: http://www.OMS.int/childgrowth/ software/en/

19. International Caries Detection and Assessment System, Coordinating Committee. Rationale and evidence for the International Caries Detection and Assessment System (ICDAS II). En: Stookey $\mathrm{G}$, editor. Proceedings of the 2005. Annual Indiana Conference, July 6-9, 2005. Indianapolis, IN: Indiana University School of Dentistry; 2006.

20. Pitts NB, Ismail AI, Martignon S, Ekstrand K, Douglas GVA, Longbottom C. ICCMS ${ }^{\mathrm{TM}}$ Guide for practitioners and educators. International Caries Classification and Management System (ICCMSTM). Implementation Workshop held June 2013. King's College London, 2014.

21. Bratthall D. Introducing the significant caries index together with a proposal for a new oral health goal for 12-year-olds, Int Dent J 2000; 50:378-84.

22. Secretaría de Salud y Bienestar Social del Municipio de Andes. Análisis de situación de salud con el modelo de los determinantes sociales de la salud. Andes: Secretaría de Salud y Bienestar Social; 2014.

23. Vasco Uribe A. Desde Heliconia hasta hoy a través del café. Medellín: Universidad de Antioquia, Departamento de Publicaciones; 1988.

24. Gobernación de Antioquia. Memorias del foro subregional ¿De qué vivirán los pobladores rurales del Suroeste? Ciudad Bolívar, 2013 [Internet]. Disponible en http://conectarural.org/sitio/sites/ default/files/mem-suroeste.pdf

25. República de Colombia, Departamento Administrativo Nacional de Estadística (DANE). Censo Nacional Agropecuario. Bogotá: DANE; 2014.

26. Colledge M. Economic cycles and health: Towards a sociological understanding of the impact of the recession on health and illness. Social Science \& Medicine. 1982; 16(22): 1919-27.

27. Ruiz-Ramos M, Córdoba JA, Bacigalupe A, Juárez S, Escolar-Pujolar A. Crisis económica al inicio del siglo XXI y mortalidad en España. Tendencia e impacto sobre las desigualdades sociales. Informe
Sespas 2014. Gaceta Sanitaria. 2014 jun.; (28) S1:89-96.

28. República de Colombia, Departamento Nacional de Planeación (DNP). Informe misión desarrollo rural. Informe detallado de la Misión para la Transformación del Campo, tomo 1. Bogotá: DNP; 2015.

29. Fundación Corona, Red de Ciudades Cómo Vamos. Informe Primera Infancia Cómo Vamos. Identificando desigualdades para impulsar la equidad en la infancia colombiana. Bogotá, 2015.

30. República de Colombia, Ministerio de Protección Social. Encuesta Nacional de la Situación Nutricional en Colombia, 2010 [Internet]. Disponible en: http://www.icbf.gov.co/portal/page/portal/ PortalICBF/Bienestar/ENSIN1/ENSIN2010/ Resumenfi.pdf

31. Hadad NLG, Del Castillo CE. Determinantes sociales de salud y caries dental. Odontol Pediatr 2001;10(1):13-9.

32. Vargas CM, Ronzio CR. Disparities in early childhood caries. BMC Oral Health. 2006; 6 (Supl. 1):S3.

33. Faggiano F, Di Stanislao F, Lemma P, Renga G. Role of social class in caries occurrence in 12 year olds in Turin, Italy. Eur J Public Health. 1999; 9:109-13.

34. Batchelor PA, Sheiham A. The distribution of burden of dental caries in school children: a critique of the high-risk caries prevention strategy for populations. BMC Oral Health. 2006; 6:1-5.

35. Ferreira SH, Beria JU, Kramer PF, Feldens EG, Feldens CA. Dental caries in 0- to 5-year-old Brazilian children: prevalence, severity, and associated factors. Int J Paediatr Dent. 2007; 17(4):289-96.

36. Pitts N, Amaechi B, Niederman R, Acevedo AM, Vianna R, Ganss C et al. Global oral health inequalities: dental caries task group--research agenda. Adv Dent Res 2011; 23(2): 211-20.

37. Bernabe E, Sheiham A, Sabbah W. Income, income inequality, dental caries and dental care levels: an ecological study in rich countries. Caries Res. 2009; 43(4):294-301.

38. World Health Organization (WHO). The World Oral Health Report 2003. Continuous improvement of oral health in the 21st century - the approach of the WHO Global Oral Health Programme. Ginebra: World Health Organization; 2003.

39. Bönecker M, Ardenghi T, Oliveira L, Sheiham A, Wagner M. Trends in dental caries in 1- to 4-yearold children in a Brazilian city between 1997 and 2008. Int J PaediatrDent. 2010; 20:125-31.

40. Peres MA, Peres KG, de Barros AJD, Victoria CG. The relation between family socioeconomic trajectories from childhood to adolescence and dental caries and associated oral behaviours. J Epidemiol Community Health. 2007; 61:141-5.

41. Antunes JL, Narvai PC, Nugent ZJ. Measuring inequalities in the distribution of dental caries. Community Dent Oral Epidemiol. 2004; 32(1):41-8.

42. Levin KA, Davies CA, Topping GVA, Assaf AV, Pitts NB. Inequalities in dental caries of 5-year-old children in Scotland, 1993-2003. European Journal of Public Health. 2009; 19(3):337-42. 
43. Piovesan, C, Mendes, FM, Ferreira, FV, Guedes, RS, Ardenghi, TM. Socioeconomic inequalities in the distribution of dental caries in Brazilian preschool children. J Public Health Dent. 2010; 70:319-26.

44. Burt BA. Concepts of risk in dental public health. Community Dent Oral Epidemiol. 2005; 33:240-7.

45. Escobar G, Ramírez BS, Franco A, Tamayo AM, Castro JF. Experiencia de caries dental en niños de 1 a 5 años de bajos ingresos. Medellín. Colombia. Rev CES Odont. 2009; 22(1):21-8.

46. Ortiz A. Condiciones de vida y de salud bucal del escolarizado y su familia, municipio de Caroni, estado Bolívar [Tesis de maestría en Odontología
Social]. Facultad de Odontología, Universidad Central de Venezuela; 1992.

47. Petersen PE, Bourgeois D, Ogawa H, EstupinánDay S, Ndiaye C. The global burden of oral diseases and risks to oral health. Bull World Health Org. 2005; 83(9):661-9.

48. Hobdell M, Petersen PE, Clarkson J, Johnson N. Global goals for oral health 2020. Int Dent J. 2003; 53(5):285-8.

49. Organización Mundial de la Salud (OMS). Subsanar las desigualdades en una generación: alcanzar la equidad sanitaria actuando sobre los determinantes sociales de la salud. Ginebra: OMS; 2008. 